\title{
Burden and correlates of atrial fibrillation among hypertensive patients attending a tertiary hospital in Tanzania
}

\author{
Smita Bhalia ${ }^{1 *} \mathbb{D}$, Pedro Pallangyo', Abuu Dalidali², Saada Salum², Richard Kawajika', Edna Kajuna ${ }^{3}$, \\ Happiness Kusiima ${ }^{1}$, Engerasiya Kifai ${ }^{1,2}$, Peter Kisenge ${ }^{1}$, Tatizo Waane ${ }^{1}$ and Mohamed Janabi ${ }^{1}$
}

\begin{abstract}
Background: Atrial fibrillation (AF) is the most common supra ventricular cardiac arrhythmia, which presents with variety of clinical symptoms. Hypertension increases risk of developing Atrial fibrillation by 1.5 fold. Together Atrial fibrillation and hypertension doubles the risk of morbidity and mortality. We aimed to determine the prevalence of AF and describe associated factors among hypertensive patients attending tertiary hospital in Tanzania.

Methods: A cross-sectional hospital-based study, involving 391 hypertensive patients visiting the Jakaya Kikwete Cardiac Institute was conducted between October to December 2017. Categorical variables were analyzed using chi square while student t- test was used to analyze continuous variables. Multivariate logistic regression analysis was performed to determine factors associated with AF. All analysis was two sided and p-value of $<0.05$ was used to be not significant.

Results: AF was detected in 40 (10.2\%) patients. Atrial fibrillation was associated with $\mathrm{BMI} \geq 25$ (OR 4.4, 95\% Cl 1.1-7.7, pvalue 0.02), ejection fraction <50\% (OR 3.0, 95\%Cl 1.1-8.2, p-value 0.03), Left Atrial diameter $>40 \mathrm{~mm}(\mathrm{OR} 9.4,95 \% \mathrm{Cl} 2.1-$ 43.2, p-value <0.01) and eGFR<60 (OR 2.9, 95\%Cl 1.1-7.8, p-value 0.04).

Conclusion: Atrial fibrillation is considerably prevalent among the hypertensive sub-population. Prompt diagnosis and timely management is vital to prevent complications and premature mortality.
\end{abstract}

Keywords: AF, Hypertension, Tanzania

\section{Background}

Hypertension is on the rise in low and middle-income countries who historically have battled communicable diseases. European society of cardiology (ESC) in 2016 reported that there were 20.9 million men and 12.6 million females living with atrial fibrillation around the world [1]. Health facilities in Tanzania are now faced with an ever growing number of hypertensive patients with multiple comorbidities including atrial fibrillation. There is a five-fold increase of atrial fibrillation in the presence of hypertension

\footnotetext{
*Correspondence: sbhaliaa@gmail.com

'Department of Adult Cardiology, Jakaya Kikwete Cardiac Institute, P. O Box 65141, Dar es Salaam, Tanzania

Full list of author information is available at the end of the article
}

and hypertension related heart diseases [2]. The link between hypertension and atrial fibrillation is unclear. Timely diagnosis of Atrial fibrillation and maintaining blood pressure at optimal levels have been shown to significantly lower morbidity and mortality among hypertensive patients. This situation calls for keener clinical assessment of hypertensive patients for timely diagnosis and management of $\mathrm{AF}$ as well as maintenance of blood pressure at target levels.

\section{Methodology}

\section{Recruitment}

A total of 491 hypertensive patients were consecutively enrolled in this Cross-sectional hospital-based study conducted at Jakaya Kikwtete Cardiac Institute. For the 
purpose of this study and after thorough literature review we developed and pre tested a questionnaire, comprising of sociodemographic, and clinical history followed by anthropometric measurement and 12 lead ECG testing using Phillips machine, echocardiography using Siemens SC300 and blood test results of lipid profile, serum creatinine and random blood sugar. Anthropometric measurement and ECG were performed by trained medical assistant and echocardiography were performed by blinded senior cardiologist.

\section{Statistical analysis}

Data analysis was done using SPSS (Statistical package for social sciences) version 20 Categorical variables were analyzed using chi square while student $t$ - test was used to analyze continuous variables. Multivariate logistic regression analysis was performed to determine factors associated with AF. All analysis was two sided and pvalue of $<0.05$ was used to be not significant.

\section{Results}

A total of 391 hypertensive patients were enrolled into the study during Oct - Dec 2017. Mean age of participants was $58 \pm 13.47$ years, $56 \%$ were male, $53.7 \%$ used alcohol and $11.3 \%$ smoked. Mean SBP, DBP and pulse rate were $150.1 \pm 28.1,85.7 \pm 18.6$ and $81.8 \pm 20.3$ respectively. $79.8 \%$ of the participants were on antihypertensive medication, $28.1 \%$ had renal insufficiency and $58.3 \%$ had anemia. General characteristics of participants are summarized in the Table 1.

The sociodemographic characteristics associated with AF among hypertensive patients at $\mathrm{JKCl}$

AF was detected in 40 (10.23\%) patients. The sociodemographic characteristics in both patients with AF and without AF group were similar and the prevalence doubled from the age 58 years and above, alcohol consumption $189(55.2 \%)$ and smoking $38(12 \%)$ as shown in Table 2.

\section{Clinical characteristics associated with atrial fibrillation among hypertensive patients at $\mathrm{JKCl}$. (N-391)}

The most common clinical presentation of participants with AF were easy fatigability (42.5\%) and palpitation (35\%) and both were statistically significant compared to those without AF. Similarly, majority of the participants with AF had reduced ejection fraction $<50 \%$ (adjusted odds ratio 4.371, $p$-value $0.021,95 \%$ CI $1.077-7.820$ ) and dilated left atrial size $\geq 40 \mathrm{~mm}$ (adjusted odds ratio 9.417, p-value $0.004,95 \% \mathrm{CI} 2.051-43.247)$ and both were found to be independent predictors of AF. Left ventricular hypertrophy was assessed on ECG and ECHO. On ECG using Sokolow- Lyon criteria 30.8\% (OR 2.05, pvalue $0.044,95 \%$ CI $1.019-4.008$ ) while on echocardiography $57.7 \%$ of the participants with $\mathrm{AF}$ and $70.1 \%$ of
Table 1 The baseline characteristics of study population at $\mathrm{JKCl}$ (N-391)

\section{Characteristics}

Sociodemographic

Age mean (SD)

$58.01(13.5)$

Sex; Male (\%)

$221(56.5)$

Female (\%)

$170(43.5)$

Marital status; Married/ Cohabiting (\%)

$301(77)$

Single/divorced/ widow/widower (\%)

$90(23)$

Education; Formal (\%)

$326(83.4)$

Informal (\%)

$65(16.6)$

Alcohol (\%)

$210(53.7)$

Smoking (\%)

$44(11.3)$

Clinical characteristics

No (\%)

Chest pain

$85(21.7)$

Easy fatigability

$93(237)$

Cough

$37(9.5)$

Awareness of heartbeat

$56(14.3)$

Shortness of breath

$121(30.9)$

Body swelling

$44(11.2)$

Cerebral vascular event

Others $^{a}$

$24(6.1)$

$121(30.9)$

Diabetes Mellitus

$92(23.5)$

Antihypertensive medication use

$312(79.8)$

Weight mean (SD) in kg

$75.41 \pm 14.5$

BMI

$28.75 \pm 5.3$

SBP mean (SD) $\mathrm{mmHg}$

$150.12 \pm 28.17$

DBP mean (SD) $\mathrm{mmHg}$

$85.71 \pm 18.60$

Pulse

$81.86 \pm 20.32$

Lab characteristics

mean $\pm S D$

Creatinine umol//

$185.41 \pm 289$

eGFR(ml/min/1.73 m2)

$77.88 \pm 36.35$

Total cholesterol (mmol/)

$5.02 \pm 1.5$

Triglyceride ( $\mathrm{mmol} / \mathrm{l})$

HDL (mmol/l)

LDL (mmol/l)

$1.7 \pm 0.88$

$1.08 \pm 0.34$

$3.3 \pm 1.3$

Anemia

$228(58.3 \%)$

ECG

AF

40 (10.23\%)

No $A F$

351 (89.77\%)

Echo $(N=265)$

IVSD

$\geq 12 \mathrm{~mm}$

$14.31 \pm 3.4$

$184(70 \%)$

LVEF (\%)

$56.6 \pm 18.8$

LAD

$56.5 \pm 18.8$

Continuous variables are presented as mean \pm standard deviation, while categorical variables are presented as proportions, number of patients, Height and weight were used to calculate body mass index (BMI weight in $\mathrm{kg} /$ height in meters ${ }^{2}$ ), SBP systolic blood pressure, DBP diastolic blood pressure, eGFR estimated glomerular filtration rate calculated using modification of diet in renal disease study equation, HDL high density lipoprotein, LDL low density lipoprotein, IVSD interventricular septal diameter, LVEF left ventricular ejection fraction, $L A D$ left atrial diameter. ${ }^{a}$ Other clinical presentation included: nausea, vomiting, fainting drug refill, follow-up clinic and referral from other clinic for investigation 
Table 2 Showing sociodemographic characteristics associated with AF among hypertensive patients $(N=391)$

\begin{tabular}{lllll}
\hline Characteristics & & $\begin{array}{l}\text { AF (\%) } \\
(N=40)\end{array}$ & $\begin{array}{l}\text { NO AF (\%) } \\
(N=351)\end{array}$ & $P$ value \\
\hline Age (years) & $<65$ & $22(55)$ & $242(68.9)$ & 0.074 \\
Sex & $\geq 65$ & $18(45)$ & $109(31.1)$ & \\
Education level & Formal education & $32(80)$ & $294(83.8)$ & 0.507 \\
Alcohol & Ever used & $17(42.5)$ & $189(53.8)$ & 0.172 \\
Smoking & Ever smoked & $3(7.3)$ & $41(11.7)$ & 0.428 \\
\hline
\end{tabular}

the participants without AF had LVH as shown in Table 3.

Logistic regression model of 15 variables was used to assess for AF associated factors. in the bivariate model out of which nine variables were found to increase probability of developing AF, however, when those nine characteristics were adjusted for confounders in a multivariate model, four characteristics i.e. (BMI $\geq 25$, $\mathrm{EF}<50 \%, \mathrm{LAD}>40 \mathrm{~mm}$ and $\mathrm{eGFR}<60 \mathrm{ml} / \mathrm{min} / 1.73 \mathrm{~m}^{2}$ were found to be independent predictors of $\mathrm{AF}$ as shown in Table 4 below.

\section{Discussion}

This study showed that the prevalence of AF in the patients visiting JKCI with hypertension is $10.23 \%$. Similar results were observed previously in both western and Asian populations, (9.75\%) Kosovo, (9.1\%) Turkey and (8\%) Brazil and slightly lower in studies conducted in Senegal (5.35\%), South Africa (4.6\%), and Thailand (3.4\%) [3-7]. the prevalence of AF in our study is likely to be a valid estimate for the Tanzanians hypertensive population. The center attends patients from the entire country tertiary center in the country serving patients with cardiovascular diseases. The preponderance of $\mathrm{AF}$ and high $\mathrm{BP}$ seen in males compared with females in our study has been reported in other studies could be due to degenerative process of atrial muscle and conducting cell [8-12].

Alcohol consumption was common among study population (42.5\%). Studies have shown that alcohol is known to produces arrythmogenic substrates thus triggering $\mathrm{AF}$ [13-15].

In our study more than half of the patients with $\mathrm{AF}$ were found to have low total cholesterol, triglyceride, HDL and high LDL, similar trend was seen in MESA, FHS and post hoc analysis of lipid lowering treatment to prevent heart attack trial (ALLHAT) $[16,17]$. however a study from Japan showed high levels of cholesterol, HDL, LDL being associated with decreased risk of AF while triglyceride were not associated with AF [18].

The most common clinical presentation of participants with AF were palpitation and easy fatigability which were significantly higher compared to no AF group similar
Table 3 Clinical presentation of patients with atrial fibrillation among Hypertensive patients $(N=391)$

\begin{tabular}{|c|c|c|c|}
\hline Presenting complain & $\begin{array}{l}\mathrm{AF} \\
\boldsymbol{N}=40(\%)\end{array}$ & $\begin{array}{l}\mathrm{NO} \text { AF } \\
\boldsymbol{N}=351(\%)\end{array}$ & $\boldsymbol{P}$ value \\
\hline Chest pain & $11(27.5)$ & $74(21.1)$ & 0.351 \\
\hline Shortness of breath & $16(40)$ & $105(29.9)$ & 0.165 \\
\hline Palpitation & $14(35)$ & $42(12)$ & $<0.001$ \\
\hline Easy fatigability & $17(\mathbf{4 2 . 5 )}$ & $76(21.7)$ & 0.003 \\
\hline Body swelling & $7(17.5)$ & $37(10.5)$ & 0.187 \\
\hline Cough & $6(15)$ & $31(8.8)$ & 0.207 \\
\hline Stroke & $4(10)$ & $20(5.7)$ & 0.856 \\
\hline Others* & $4(10)$ & $117(33.3)$ & 0.002 \\
\hline DM & $5(12.5)$ & $87(24.8)$ & 0.83 \\
\hline $\mathrm{SBP} \geq 140 \mathrm{mmHg}$ & $21(52.5)$ & $244(69.5)$ & 0.029 \\
\hline $\mathrm{DBP} \geq 90 \mathrm{mmHg}$ & $11(27.5)$ & $202(57.5)$ & 0.068 \\
\hline \multicolumn{4}{|l|}{ Heart rate } \\
\hline$\geq 100 \mathrm{~b} / \mathrm{m}$ & $8(20)$ & $48(13.7)$ & 0.189 \\
\hline $99-60 \mathrm{~b} / \mathrm{m}$ & $25(62.5)$ & $265(75.5)$ & 0.087 \\
\hline$<59 \mathrm{~b} / \mathrm{m}$ & $7(17.5)$ & $37(10.5)$ & 0.279 \\
\hline \multicolumn{4}{|c|}{ Biochemical characteristics } \\
\hline Total Cholesterol & $1.45 \pm 0.5$ & $1.47 \pm 0.5$ & \\
\hline$\geq 5.2 \mathrm{mmol} / \mathrm{l}$ & $18(45)$ & $174(49.6)$ & 0.584 \\
\hline$<5.2 \mathrm{mmol} / \mathrm{l}$ & $22(55)$ & $177(50.4)$ & \\
\hline Triglyceride & $1.61 \pm 0.8$ & $1.7 \pm 0.88$ & \\
\hline$<1.69 \mathrm{mmol} / \mathrm{l}$ & $27(67.5)$ & $214(61)$ & 0.421 \\
\hline$\geq 1.69 \mathrm{mmol} / \mathrm{l}$ & $13(32.5)$ & $137(39)$ & \\
\hline $\mathrm{HDL}$ & $1.00 \pm 2.92$ & $1.08 \pm 0.3$ & \\
\hline$\geq 1.04 \mathrm{mmol} / \mathrm{l}$ & $16(40)$ & $174(49.6)$ & 0.251 \\
\hline$<1.039 \mathrm{mmol} / \mathrm{l}$ & $24(60)$ & $177(50.4)$ & \\
\hline LDL & $3.2 \pm 1.26$ & $3.3 \pm 1.3$ & \\
\hline$<3.39 \mathrm{mmol} / \mathrm{l}$ & $17(42.5)$ & $190(54.1)$ & 0.163 \\
\hline$\geq 3.4 \mathrm{mmol} / \mathrm{l}$ & $23(57.5)$ & $161(45.9)$ & \\
\hline BMI(N-377) & $29.53 \pm 5.25$ & $28.82 \pm 5.21$ & \\
\hline$\geq 25$ & $30(83.3)$ & $228(66.9)$ & 0.043 \\
\hline Egfr & $65.95 \pm 31.20$ & $79.24 \pm 36.3$ & \\
\hline$<60$ & $17(42.5)$ & $93(26.5)$ & 0.033 \\
\hline \multicolumn{4}{|l|}{ ECG } \\
\hline LVH (Sokolow) & $14(35)$ & $73(20.8)$ & 0.041 \\
\hline \multicolumn{4}{|l|}{ Echo $N=263$} \\
\hline$E F \geq 50$ & $8(30.8)$ & $171(69.5)$ & $<0.001$ \\
\hline$<50$ & $18(69.2)$ & $75(30.5)$ & \\
\hline IVSD $<12 \mathrm{~mm}$ & $11(42.3)$ & $72(29.9)$ & 0.193 \\
\hline$\geq 12 \mathrm{~mm}$ & $15(57.7)$ & $169(70.1)$ & \\
\hline $\mathrm{LAD}<40 \mathrm{~mm}$ & $2(8)$ & $129(54)$ & $<0.001$ \\
\hline$\geq 40 \mathrm{~mm}$ & $23(92)$ & $110(46)$ & \\
\hline
\end{tabular}

*represents fever and diarrhea

$S B P$ systolic blood pressure, DBP diastolic blood pressure, Cholesterol $>5.2$ $\mathrm{mmol} / \mathrm{I}$ was raised, triglyceride $>1.69 \mathrm{mmol} / \mathrm{l}$ raised, $\mathrm{hdl}<1.04 \mathrm{mmol} / \mathrm{l}$ low, Idl $3.4 \mathrm{mmol} / / \mathrm{l}$ raised were identified as dyslipidemia. Reduced ejection fraction was defined as $\mathrm{EF}<50 \%$, normal $\geq 50 \%$, $\mathrm{LVH}$ on echocardiography was seen if IVSD was $\geq 12 \mathrm{~mm}$, dilated left atrial diameter $>40 \mathrm{~mm}$ 
Table 4 Logistic Regression analysis to determine factors associated with AF

\begin{tabular}{|c|c|c|c|c|c|c|}
\hline Variable & Odds ratio & $P$ value & $95 \% \mathrm{Cl}$ & Adjusted odd ratio & $95 \% \mathrm{Cl}$ & $P$ value \\
\hline Age $\geq 65 \mathrm{yr}$ & 1.817 & 0.077 & $0.936-3.524$ & - & & \\
\hline Male & 1.045 & 0.895 & $0.540-2.026$ & - & & \\
\hline $\mathrm{HDL} \geq 1.04 \mathrm{mmol} / \mathrm{l}$ & 1.475 & 0.253 & $0.757-2.871$ & - & & \\
\hline $\mathrm{LDL}<3.4 \mathrm{mmol} / \mathrm{l}$ & 1.597 & 0.165 & 0.824-3.093 & - & & \\
\hline $\mathrm{SBP}>140 \mathrm{mmHg}$ & 0.485 & 0.032 & $0.250-0.939$ & - & & \\
\hline $\mathrm{DBP}>90 \mathrm{mmHg}$ & 0.514 & 0.072 & $0.249-1.062$ & - & & \\
\hline Alcohol use & 0.605 & 0.1312 & $0.312-1.172$ & - & & \\
\hline Cigarette Smoking & 0.613 & 0.432 & $0.181-2.078$ & & & \\
\hline Diabetes Mellitus & 0.246 & 0.022 & $0.074-0.818$ & - & & \\
\hline $\mathrm{BMI} \geq 25$ & 2.478 & 0.049 & $1.002-6.126$ & 4.371 & $1.077-7.820$ & 0.021 \\
\hline$E F<50 \%$ & 5.130 & $<0.001$ & $2.136-12.318$ & 3.013 & $1.106-8.21$ & 0.031 \\
\hline $\mathrm{LAD}>40 \mathrm{~mm}$ & 13.486 & 0.001 & $3.110-58.487$ & 9.417 & $2.051-43.247$ & 0.004 \\
\hline IVSD & 0.581 & 0.197 & $0.254-1.326$ & - & & \\
\hline LVH on ECG & 2.051 & 0.044 & $1.019-4.008$ & - & & \\
\hline $\mathrm{eGFR}<60 \mathrm{ml} / \mathrm{min} / 1.73 \mathrm{~m}^{2}$ & 2.050 & 0.036 & $1.049-4.008$ & 2.902 & 0.035 & $1.077-7.820$ \\
\hline
\end{tabular}

pattern was observed in Kenya at Aga Khan University Hospital, Nairobi looking at clinical characteristics and outcomes of atrial fibrillation and flutter [19].

More than half of the participants with AF had their systolic blood pressure $140 \mathrm{mmHg}$ and normal pulse rate with majority of them on antihypertensive medication, most common groups of medication used were $(60 \%)$ ACEI/ARB, (70\%) diuretics and (55\%) beta-blockers. As shown in the study patients with AF presented with symptoms of palpitation and are more likely to receive beta blocker in the combination of their antihypertensive medication thus rate controlled AF. In the current study it was also observed that atrial fibrillation was more common among those with dilated left atrial and ejection fraction $<50 \%$ which supports the pathogenesis theory of atrial fibrillation.

Results of our study have some clinical implications. Our prevalence estimate of AF $10.25 \%$ in hypertensive is high considering the chronic course of this disease that might cause serious thromboembolic stroke. Our study has demonstrated that there are other factors that could even further increase prevalence of AF for example, males older than 58 years of age with BP $\geq 140 / 80 \mathrm{mmHg}$. Awareness and education on regular monitoring and compliance may help reduce the number of serious strokes related to $\mathrm{AF}$ by giving stroke prophylaxis treatment.

This study was not short of limitations. For instance, owing to the cross section nature of this study both predictor and outcomes variable were measured simultaneously and thus our findings cannot infer causality. Furthermore, as this study was conducted in a tertiary level hospital, referral filter bias is probable. The strength of this study it was conducted at tertiary center where patients from all over Tanzania are referred for advanced management thus the sample is representative of the whole nation.

\section{Conclusion}

Prevalence of AF in hypertensive population was found to be high. We recommend every hypertensive patient to have 12 lead ECG for early recognition of AF and prompt management.

\section{Supplementary information}

Supplementary information accompanies this paper at https://doi.org/10. 1186/s12872-020-01517-x.

\section{Additional file 1.}

Additional file 2 .

\section{Abbreviations}

AF: Atrial fibrillation; ECG: Electrocardiography; SBP: Systolic blood pressure; DBP: Diastolic blood pressure; LDL: Low density lipoprotein; HDL: High density lipoprotein; JKCl: Jakaya Kikwete cardiac institute; BP: Blood pressure; LAD: Left atrial diameter; BMI: Body mass index; EF: Ejection fraction; EGFR: Estimated glomerular filtration rate; OR: Odds ratio; ESC: European society of cardiology; SPSS: Statistical package for social sciences; ACEl: Angiotensin converting enzyme inhibitor; ARB: Angiotensin receptor blocker; Cl: Confidence interval; LVH: Left ventricular hypertrophy; IVSD: Intraventricular septal defect

\section{Acknowledgements}

Sincere thanks to all JKCl staff and patients for their kind support and cooperation during data collection.

\section{Authors' contributions}

SB and MJ conceived the study. SB performed all the data collection and entry. SB and PP performed the analysis and developed the first draft of the manuscript. EK, PK and TW performed the detailed echocardiography. AD and SS performed the ECGs. RK and HK interpreted the ECGs. EK took all the 
participant's samples for investigation. All authors contributed to this latest version of the manuscript and agreed on its submission.

\section{Funding}

Self-funded.

\section{Availability of data and materials}

The final version of Data set supporting the findings of this paper is submitted together with this manuscript to the editorial committee. All the raw data is included in this manuscript.

\section{Ethics approval and consent to participate}

Ethical clearance to carry out this study was obtained from the Research and Publications Committee of Muhimbili University of Health and Allied Sciences (MUHAS), and permission to conduct the study was sought from the Director of research, training and consultancy of Jakaya Kikwete Cardiac Institute (JKCl). All participants (or caretakers) provided written consent to participate prior to enrolment.

\section{Consent for publication}

Not applicable.

\section{Competing interests}

The authors declare that they have no competing interest.

\section{Author details}

'Department of Adult Cardiology, Jakaya Kikwete Cardiac Institute, P. O Box 65141, Dar es Salaam, Tanzania. ${ }^{2}$ Department of Clinical Support Services, Jakaya Kikwete Cardiac Institute, P. O Box 65141, Dar es Salaam, Tanzania. ${ }^{3}$ Department of Nursing, Jakaya Kikwete Cardiac Institute, P. O Box 65141, Dar es Salaam, Tanzania.

Received: 9 January 2020 Accepted: 10 May 2020

Published online: 19 May 2020

\section{References}

1. Kirchhof P, Benussi S, Kotecha D, Ahlsson A, Atar D, Casadei B, et al. 2016 ESC guidelines for the management of atrial fibrillation developed in collaboration with EACTS. Europace. 2016;18(11):1609-78.

2. Lloyd-jones DM, Wang TJ, Leip EP, Larson MG, Levy D, Vasan RS, et al. Lifetime risk for development of atrial fibrillation the framingham heart study. Circulation. 2004;110:1042-7.

3. Sliwa K, Carrington MJ, Klug E, Opie L, Lee G, Ball J, et al. Predisposing factors and incidence of newly diagnosed atrial fibrillation in an urban African community: insights from the heart of Soweto study. Heart. 2010; 96(23):1878-82.

4. Krittayaphong R, Rangsin R, Thinkhamrop B, Hurst C, Rattanamongkolgul S, Sripaiboonkij N, et al. Prevalence and associating factors of atrial fibrillation in patients with hypertension: a nation-wide study. BMC Cardiovasc Disord. 2016;16(1):1-7.

5. Mbaye A, Pessinaba S, Bodian M, Ndiaye Mouhamadou B, Mbaye F, Kane A, et al. Atrial fibrillation, frequency, etiologic factors, evolution and treatment in a cardiology department in Dakar, Senegal. Pan Afr Med J. 2010;6:16.

6. Elezi S, Qerkini G, Bujupi L, Shabani D, Bajraktari G. Management and comorbidities of atrial fibrillation in patients admitted in cardiology service in Kosovo-a single-center study. Anadolu Kardiyol Derg. 2010;10(1):36-40.

7. Karacaglar E, Atar I, Yetis B, Corut H, Ersoy B, Yilmaz K, et al. The frequency of embolic risk factors and adequacy of anti-embolic treatment in patients with atrial fibrillation: a single tertiary center experience. Anadolu Kardiyol Derg. 2012;12:384-90.

8. Gutierrez C, Blanchard DG. Atrial fibrillation: diagnosis and treatment. Am Fam Physician. 2011;83(1):61-8.

9. $\quad$ Aizer A, Fuster V. Atrial Fibrillation; ACP Medicine; 2003.

10. Matchar D, McCrory D, Pritchett EL. Management of atrial fibrillation. Ann Intern Med. 1994;121(6):1264-71.

11. Benjamin EJ, Wolf PA, D'Agostino RB, Silbershatz H, Kannel WB, Levy D. Impact of atrial fibrillation on the risk of death: the Framingham heart study. Circulation. 1998;98(10):946-52.

12. Schnabel RB, Sullivan LM, Levy D, Pencina MJ, Massaro JM, D'Agostino RB, et al. Development of a risk score for atrial fibrillation (Framingham heart study): a community-based cohort study. Lancet. 2009;373(9665):739-45.
13. Voskoboinik A, Prabhu S, Ling LH. Alcohol and atrial fibrillation: a sobering review. J Am Coll Cardiol. 2016;68(23):2567-76.

14. Samokhvalov AV, Irving HM, Rehm J. Alcohol consumption as a risk factor for atrial fibrillation: a systematic review and meta-analysis. Eur J Cardiovasc Prev Rehabil. 2010;17(6):706-12.

15. Larsson SC, Drca N, Wolk A. Alcohol consumption and risk of atrial fibrillation: a prospective study and dose-response meta-analysis. J Am Coll Cardiol. 2014;64(3):281-9.

16. Lopez FL, Agarwal SK, MacLehose RF, Soliman EZ, Sharrett AR, Huxley RR, et al. Blood lipid levels, lipid-lowering medications, and the incidence of atrial fibrillation :the atherosclerosis risk in communities study. Circ Arrhythm Electrophysiol. 2012;5(1):155-62.

17. Alonso A, Yin X, Roetker NS, Magnani JW, Kronmal RA, Ellinor PT, et al. Blood lipids and the incidence of atrial fibrillation: the multi-ethnic study of atherosclerosis and the Framingham heart study. J Am Heart Assoc. 2014; 3(5):e001211.

18. Hill H. Fixing teacher professional development. Phi Delta Kappan. 2009;90: 470-6.

19. Shavadia J, Yonga G, Mwanzi S, Jinah A, Moriasi A, Otieno H. Clinical characteristics and outcomes of atrial fibrillation and flutter at the Aga Khan University Hospital, Nairobi. Cardiovasc J Afr. 2013;24(2):6-9.

\section{Publisher's Note}

Springer Nature remains neutral with regard to jurisdictional claims in published maps and institutional affiliations.
Ready to submit your research? Choose BMC and benefit from:

- fast, convenient online submission

- thorough peer review by experienced researchers in your field

- rapid publication on acceptance

- support for research data, including large and complex data types

- gold Open Access which fosters wider collaboration and increased citations

- maximum visibility for your research: over $100 \mathrm{M}$ website views per year

At $\mathrm{BMC}$, research is always in progress.

Learn more biomedcentral.com/submissions 